\title{
Taming the swirl of self-structured liquid crystal q-plates
}

\author{
Nina Kravets and Etienne Brasselet \\ Univ. Bordeaux, CNRS, LOMA, UMR 5798, F-33400 Talence, France \\ E-mail: etienne.brasselet@u-bordeaux.fr
}

Received 1 November 2019, revised 17 December 2019

Accepted for publication 20 January 2020

Published 5 February 2020

\begin{abstract}
Spontaneously formed liquid crystal topological defects under external fields offer a natureassisted route to the creation of geometric phase optical vortex generators ( $q$-plates). Here we report on the consequences of the unavoidable swirled transverse spatial distribution of the optical axis of such optical elements on the beam shaping and we propose a swirl-compensation scheme based on the arithmetic of geometric phase optical elements.
\end{abstract}

Keywords: optical vortices, liquid crystal defects, geometric phase

(Some figures may appear in colour only in the online journal)

\section{Introduction}

In wave physics, the concept of twisted waves is closely related with phase singularities defined as locations of null amplitude where the phase is undefined and around which the phase winds by an integer multiple of $2 \pi$ are along a closed circuit. Phase singularities are actually a natural ingredient of real-world fields once recalled that three plane waves are enough to generate them. In fact, one can even make them appear as the backbone of propagating waves by choosing the Laguerre-Gauss modes as the exact basis for the solutions of the paraxial scalar Helmholtz equation. Indeed, the complex amplitude of each of these modes is proportional to the pure phase factor $\exp (i \ell \phi)$ where $\ell$ integer is the topological charge of the singularity and $\phi$ is the polar angle in the transverse plane with respect to the propagation direction. The connection between propagating beams carrying phase singularities (i.e. vortex beams) and orbital angular momentum, which has been established for light [1], sound [2] and electrons [3], led to the advent of a plethora of devices enabling the creation of vortex beams.

A straightforward solution to twist a wavefunction consists of the use of transmissive plates having azimuthally varying thickness, which impart an azimuthally varying phase profile $\Phi=\ell \phi$ to the incident field. In optics, such an approach has been experimentally reported in the early 1970s [4] and seemingly rediscovered two decades later [5]. Nowadays, much attention is paid to the development of flat-optics devices, ideally endowed with photonic function and operating wavelength that are independently adjustable. Electrically addressable liquid crystal spatial light modulators represent a popular versatile solution. Such devices allow pixel-by-pixel control of the 'dynamic' phase associated with the average optical path length of individual pixels made of uniformly oriented liquid crystals. Another kind of liquid crystal device, to which the present work is devoted, relies on the 'geometric' phase associated with the inplane orientation of the liquid crystals according to a generic approach anticipated by Bhandari [6]. The latter approach relies on a slab made of a half-wave birefringent retarder whose optical axis orientation angle, $\psi$, varies spatially. As the polarization degrees of freedom couple to the spatial ones [7], a phase profile $\Phi=2 \sigma \psi$ is imparted to an incident circularly polarized light as its helicity $\sigma= \pm 1$ is flipped ( $\sigma \rightarrow-\sigma$ ).

Since the first report of a liquid crystal geometric phase optical vortex generator [8], both the technology and its application prospects have much evolved, as recently reviewed in [9]. In particular, the so-called $q$-plate design, which refers to $\psi=q \phi$, enables the generation of phase singularities with topological charge $\ell= \pm 2 q$ and userfriendly tunable operating wavelength owing to electrically controlled half-wave retardation condition [10]. Electrically tunable operating wavelength, however, comes at the expense of a loss of patterning resolution compared to the state-of-theart standards associated with static material vortex patterns with maintained topology down to micrometer scales [11].

One decade ago, the self-structuring capabilities of liquid crystals to form topological defects behaving as geometric phase optical vortex generators [12] suggested a natureassisted route to mitigate the latter technological issue. 


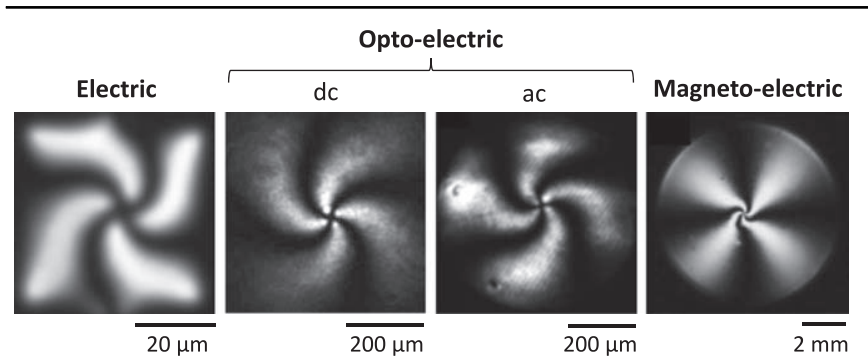

Figure 1. Images of various kinds of pinned swirled self-structured umbilical liquid crystal $q$-plate, with $q=+1$, observed between crossed linear polarizers. (Left) Electrically induced umbilic inside a square-shaped pixel with $50 \mu \mathrm{m}$ side [19]. (Middle) Opto-electrically induced umbilics using photo-activated polymer interface under dc applied voltage [17] and photoconductor substrate under ac applied voltage [20]. (Right) Magneto-electrically induced umbilic using a ring magnet with $8 \mathrm{~mm}$ diameter clear aperture [18]. In all cases, a voltage drop of a few volts across the liquid crystal layer is enough to fulfill the half-wave retardance requirement for visible light.

Different kinds of topological defects spontaneously appearing in nematic liquid crystal slabs have been identified as electrically tunable $q$-plates: umbilics [13] and disclinations [14]. Until now, it is the umbilical defects associated with $q=+1$ that have attracted the most attention and optical vortex generation with high-purity has been reported in various situations ${ }^{1}$. Indeed, several pinning strategies have been proposed to fix them in place, hence getting rid of previous limitations associated to random location of the defects [13] while preserving electrical tuning capabilities. Namely, boundary-assisted electrical pinning using pixelated electrodes [15], optically-assisted electrical pinning using photoresponsive substrates under ac [16] or dc [17] electric fields, and magnetic pinning using static ring magnets [18]. All these works offer a palette of clear aperture ranging typically over three decades from microscopic to macroscopic scales, as illustrated in figure 1.

Remarkably, swirled umbilical defects (either right-handed or left-handed) are observed independently of the pinning strategy, whose origin lies in the anisotropic elasticity of liquid crystals as discussed in detail in [21, 22]. Still, we notice that the appearance of swirled nematic textures goes beyond the particular case of umbilics as it is a generic feature of elastic energy minimization in confined geometries, such as spherical droplets, circular sessile droplets and tactoids, as discussed in [23]. In the framework of optical vortex generation, swirled designs are associated to geometric phase lensing effects, which can be an asset, for instance for longitudinal splitting of circularly polarized components of a multi-vortex field [24], or a drawback, for instance in vortex Fourier filtering [25]. This motivates the development of agile swirl-control strategies, which is the purpose of the present work. Hereafter, we start in section 2 by presenting the used umbilical $q$-plate (UQP) and characterizing quantitatively its swirl. The effects of the swirl on light is addressed in section 3 and in section 4 we introduce a swirl-compensation

\footnotetext{
1 Maximal purity equal to 1 corresponds to half-wave retardance condition for the used wavelength.
}

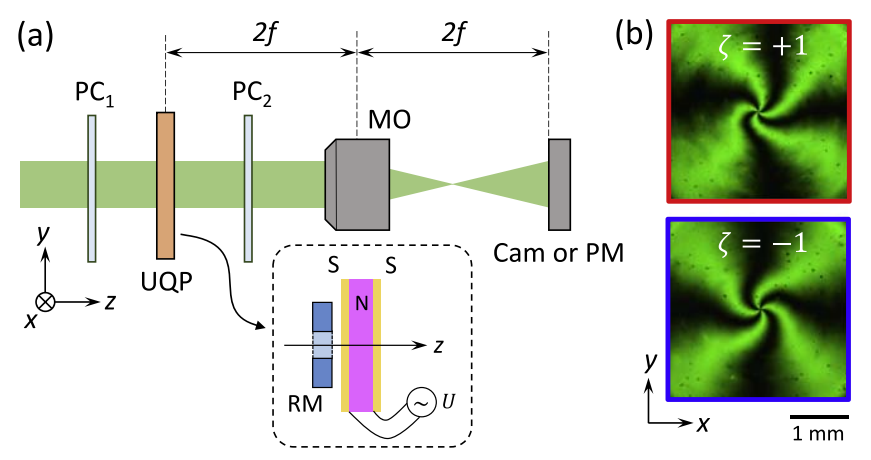

Figure 2. (a) Experimental setup used for the characterization of swirled UQP generated by magneto-electric stimulus. $\mathrm{PC}_{1,2}$ : polarization controllers; Cam: camera; PM: powermeter; MO: microscope objective with $4 \times$ magnification, numerical aperture $\mathrm{NA}=0.1$ and effective focal length $f=45 \mathrm{~mm}$. The inset shows the detail of the magneto-electric UQP. RM: ring magnet; S: glass substrates with transparent electrodes; N: nematic liquid crystal film. (b) Oppositely swirled umbilics observed between crossed linear polarizers using incoherent illumination.

scheme based on the arithmetic of geometric phase optical elements.

\section{Magneto-electric swirled UQP}

For our experiments, we choose an UQP produced according to the magneto-electric approach described in [18], which has the advantage to combine robustness, simplicity of use, up to centimeter-size clear aperture and spectrally agile behavior. The schematic of the UQP and setup used for its characterization is shown in figure 2 . We use of a $20 \mu \mathrm{m}$ thick film of nematic liquid crystal (MLC-6608 from Merck Japan) sandwiched between two parallel glass substrates provided with indium-tin oxide electrodes and spin-coated with a polyimide anchoring layer ensuring perpendicular alignment of the liquid crystal orientation at both ends of the film. In the absence of external fields, the liquid crystal orientation is therefore perpendicular to the sample plane everywhere in the bulk of the film, which defines the $z$ axis; see figure 2(a). The operating wavelength is $\lambda=532 \mathrm{~nm}$ and experiments are carried out at $20^{\circ} \mathrm{C}$.

The static magnetic field is ensured by a nickel-plated neodynium (grade N50) ring magnet whose magnetization is directed along the $z$ axis. Its thickness, inner diameter and outer diameter are respectively $6 \mathrm{~mm}, 4 \mathrm{~mm}$ and $12 \mathrm{~mm}$ and it is associated with a pull force of $32 \mathrm{~N}$ (manufacturer data sheet). The front side of the magnet is placed at $\sim 2 \mathrm{~mm}$ distance from the liquid crystal layer. Since the nematic has a negative dielectric anisotropy $\epsilon_{a}=-4.2$ at $1 \mathrm{kHz}$ frequency, the liquid crystal is expected to reorient above the electrical reorientation threshold voltage amplitude given by $U_{\text {th }}=$ $\pi \sqrt{K_{3} /\left(\epsilon_{0}\left|\epsilon_{a}\right|\right)}=2.19 \mathrm{~V}$ when using a square waveform, where $\epsilon_{0}$ is the vacuum permittivity. Consistently, we detect the liquid crystal reorientation above $U_{\text {th }}$, which leads to the formation of an umbilic, spontaneously swirled in a random direction. This is illustrated in figure 2(b) that displays images of oppositely swirled umbilics (labeled with the index 
(a)

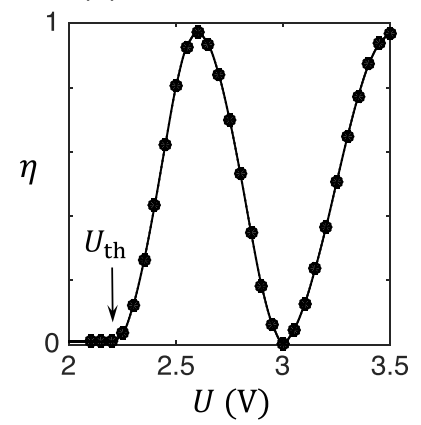

(b)

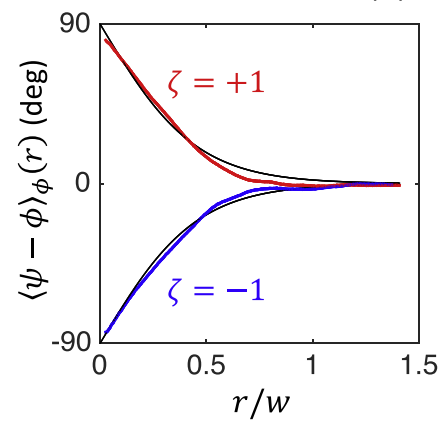

Figure 3. (a) Typical voltage dependence of the purity $\eta$ of the optical vortex generation process of a swirled umbilic. (b) Azimuth average radial profile of the liquid crystal in-plane orientation for $\zeta= \pm 1$ (red and blue curves) at $U=2.60 \mathrm{~V}$. The black curves refer to the fit by the function $\zeta \varphi(r)=(\zeta \pi / 2)[1-\tanh (r / R)]$ with $R=450 \mu \mathrm{m}$.

$\zeta= \pm 1)$ when observed between crossed linear polarizers (oriented along the $x$ and $y$ axis throughout the paper), under an applied voltage $U=2.60 \mathrm{~V}$, which corresponds to the half-wave retardance condition. This corresponds to the full conversion of a circularly polarized input light with helicity $\sigma$ into a contra-circularly polarized output light having opposite helicity $-\sigma$.

Experimentally, we use either incoherent illumination from a halogen lamp spectrally filtered at $532 \mathrm{~nm}$ wavelength or a collimated Gaussian laser beam having a beam waist radius $w \simeq 400 \mu \mathrm{m}$ at the liquid crystal plane, propagating along the $z$. The light passes through two polarization controllers ( $\mathrm{PC} 1$ and $\mathrm{PC} 2$ ), each being a quarter-wave plate coupled with a wire-grid polarizer. This allows us to prepare the input polarization state and selecting on-demand the projection of the output light onto the desired polarization state.

The characterization of the UQP is made in two steps. First, using a circularly polarized input laser beam, we determine the purity parameter $\eta=P_{-\sigma} /\left(P_{-\sigma}+P_{+\sigma}\right)$, where $P_{ \pm} \sigma$ is the power of the circularly polarized component of the output beam with helicity $\pm \sigma$, as a function of the applied voltage. A typical result is shown in figure 3(a), which does not exhibit a noticeable influence of the swirl handedness. In our study, we further use three values of voltage that correspond to quarter-wave $(\eta=0.5)$, half-wave $(\eta=1)$ and fullwave $(\eta=0)$ retardance conditions, namely, $U=2.42 \mathrm{~V}$, $U=2.60 \mathrm{~V}$ and $U=3.00 \mathrm{~V}$, respectively. Second, we retrieve quantitatively the radial distribution of the effective in-plane orientation angle of the optical axis of the UQP, $\psi(r, \phi)$. This is done from spatially resolved Stokes polarimetry of the light emerging from the liquid crystal sample, whose recipe can be found in [20]. Assuming an axisymmetric description of the swirled structure of the form

$$
\psi(r, \phi)=\phi+\zeta \varphi(r),
$$

we determine the radial dependence of the swirl function according to $\zeta \varphi(r)=\langle\psi-\phi\rangle_{\phi}(r)=\frac{1}{2 \pi} \int_{0}^{2 \pi}[\psi(r, \phi)-\phi] d \phi$.

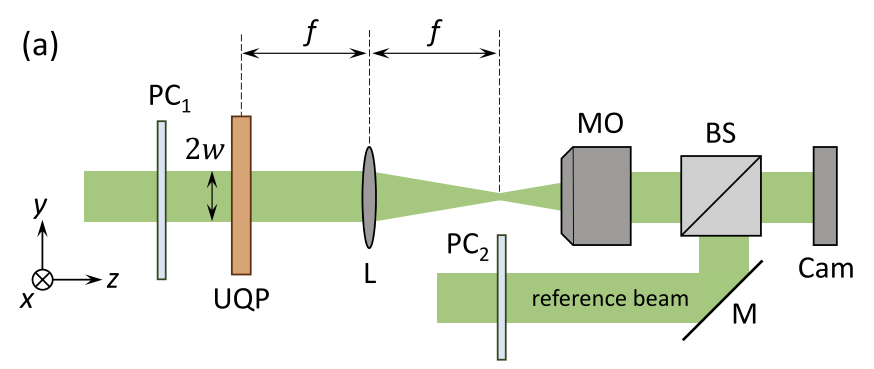

(b)

(b) $\zeta=+1$
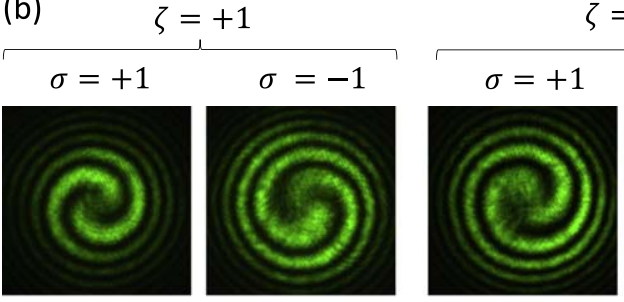

$=-1$
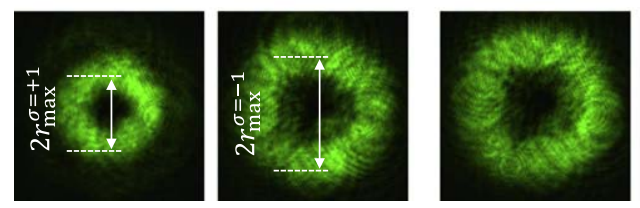

$\sigma=-1$

Figure 4. (a) Setup for analyzing the far-field of the generated vortex field, at $U=2.60 \mathrm{~V}$, as a function of the swirl handedness $\zeta$ and the helicity $\sigma$ of the incident beam. L: spherical lens with focal length $f=200 \mathrm{~mm}$; M: mirror; BS: beamsplitter; MO: microscope

objective with $10 \times$ magnification, numerical aperture NA $=0.25$.

The incident light field is a collimated circularly polarized Gaussian laser beam with helicity $\sigma$ and waist radius $\simeq 400 \mu \mathrm{m}$ in the plane of the sample. (b) (Top) Spiraling interference pattern using a coaxial Gaussian reference beam. (Bottom) Far-field intensity profiles reimaged by the microscope objective on the camera.

The results are shown in figure 3(b) for $\zeta= \pm 1$ (color curves) where the black curves refer to the fit with the following ansatz

$$
\varphi(r)=\frac{\pi}{2}[1-\tanh (r / R)],
$$

where the characteristic length $R$ is the only adjustable parameter, and we find $R \simeq 450 \mu \mathrm{m}$.

\section{Swirl effects on processed light}

Using the setup sketched in figure 4(a), we explore the farfield phase and intensity distributions of the generated optical vortices. The idea is to analyze the light in the focal plane of the lens $\mathrm{L}$ with focal length $f=200 \mathrm{~mm}$ by re-imaging it onto the camera owing to the microscope objective MO. Firstly, we note that the occurrence of the double-charge onaxis phase singularity with $\sigma$-dependent sign is unaffected by the swirl handedness; see the top row of figure 4(b). It is an expected result, because the swirl is not affecting the topology of the UQP. In contrast, the far-field intensity distributions exhibit a substantial dependence on the product $\zeta \sigma= \pm 1$; see the bottom row of figure $4(b)$.

To understand the latter point, let us stress the fact that the far-field intensity distribution of two light fields of the form $E_{ \pm}(r, \phi)=E_{0}(r) \exp [i \ell \phi \pm i \Psi(r)]$, with $\ell$ integer and 


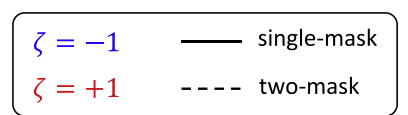

(a)

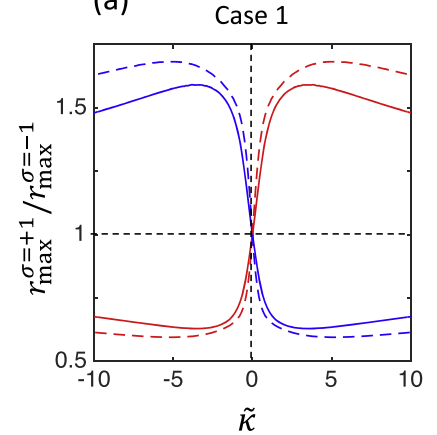

(b)

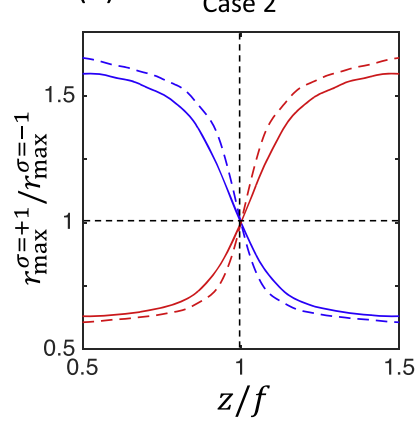

Figure 5. Ratio between the radii of maximal intensity of the doughnut intensity profiles, namely, $r_{\max }^{\sigma=+1} / r_{\max }^{\sigma=-1}$ for $\zeta= \pm 1$, taking the experimental values $f=200 \mathrm{~mm}$ and $w=400 \mu \mathrm{m}$, see figure 4(a). (a) Case 1: in the focal plane of the lens L as a function of the normalized curvature $\tilde{\kappa}$ of the incident Gaussian beam with waist $w$ in the plane of the sample, where $\tilde{\kappa}=\kappa z_{0}$ with $z_{0}=\pi w^{2} / \lambda$. The chosen typical range $|\tilde{\kappa}|<10$ for the normalized curvature is dictated by the preservation of the paraxial approximation. (b) Case 2: around the focal plane of the lens $\mathrm{L}, z$ being the distance from the lens and assuming an incident Gaussian beam with planar wavefront. The single-mask situation refers to section 3 , whereas the two-mask situation refers to section 4.2 .

$E_{0}(r)$ the field magnitude (positive real), are identical. Indeed, let us consider the Fourier transform of $E_{ \pm}$in the polar coordinate system $(\chi, \theta)$ as $\tilde{E}_{ \pm}(\chi, \theta)=\int_{0}^{2 \pi}$ $\int_{0}^{\infty} E_{ \pm}(r) \exp [-i \chi r \cos (\theta-\phi)] r d r d \phi$. The corresponding intensity profile is axisymmetric and is given by $I_{ \pm}(\chi, \theta) \propto\left|\int_{0}^{\infty} E_{0}(r) J_{\ell}(\chi r) e^{ \pm} i \Psi(r) r d r\right|^{2}$, where $J_{\ell}$ is the $\ell$ th-order Bessel function of the first kind, and one gets $I_{+}=I_{-}$. A possible reason of the experimentally observed discrepancy is related to the additional radial-coordinatedependent phase factor, e.g. intrinsic curvature of the Gaussian beam. This is numerically illustrated in two cases in what follows, based on analytical formulations derived from [26] and using the swirl ansatz given by equation (2).

Case1. Let us model the incident laser Gaussian field as $\left.E_{\text {in }}^{(1)}(r)=E_{0} \exp \left[-\frac{r^{2}}{w^{2}}-\frac{i \pi r^{2}}{\lambda} \kappa\right)\right]$ where $\kappa$ is the incident beam curvature. By modeling the UQP as a mask with complex transmittance $\exp [2 i \sigma \psi(r, \phi)]$, the output field $E_{\text {out }}$ in the focal plane of the lens $\mathrm{L}$ is thus expressed as

$$
\begin{aligned}
E_{\text {out }}^{(1)}(r, \phi) \propto & E_{0} \frac{\mathrm{e}^{2 i \sigma \phi}}{\lambda f} \int_{0}^{\infty} J_{2}\left(\frac{2 \pi \rho r}{\lambda f}\right) e^{-\frac{\rho^{2}}{w^{2}}+2 i \sigma \zeta \varphi(\rho)} \\
& \times e^{-i \frac{\pi \rho^{2}}{\lambda} \kappa} \rho d \rho .
\end{aligned}
$$

From the above equation, we calculate the ratio between the radii of maximal intensity of the doughnut intensity profiles for $\sigma=+1$ and $\sigma=-1$, as defined in figure 4(b). The results are displayed as solid curves in figure 5(a) for $\zeta= \pm 1$. Identical doughnut intensity profile are obtained only for $\kappa=0$, which corresponds to a planar incident wavefront, whereas any deviation from this ideal setting leads to a ratio close to that observed in the experiments, which offer a decent explanation to the observations reported in figure 4(b).

Case2. From an experimental point of view, it is also interesting to evaluate the role of a mismatch between the focal plane of the lens $\mathrm{L}$ and the observation plane. This is done by considering an incident beam with a planar wavefront, $E_{\text {in }}^{(2)}(r)=E_{0} \exp \left(-r^{2} / w^{2}\right)$ and evaluating the output field $E_{\text {out }}$ at a distance $z$ from the lens L, which gives

$$
\begin{aligned}
E_{\text {out }}^{(2)}(r, \phi) \propto & E_{0} \frac{\mathrm{e}^{2 i \sigma \phi}}{\lambda f} \int_{0}^{\infty} J_{2}\left(\frac{2 \pi \rho r}{\lambda f}\right) \mathrm{e}^{-\frac{\rho^{2}}{w^{2}}+2 i \sigma \zeta \varphi(\rho)} \\
& \times e^{-i \frac{\pi \rho^{2}}{\lambda}\left(\frac{z-f}{z f}\right)} \rho d \rho,
\end{aligned}
$$

where one identifies $(z-f) /(z f)$ as the $z$-dependent curvature in the last term of the integrand. The results are displayed as solid curves in figure 5(b) and, as expected, the swirl has no effect in the focal plane of the lens. Indeed, equation (3) for $\kappa=0$ and equation (4) for $z=f$ are identical. Although the extremal values for the calculated ratio explore the same range in cases 1 and 2, we note that our experimental precision when positioning the lens $\mathrm{L}$, the reimaging microscope objective MO and the camera are of the order of less than a millimeter. Therefore, present analysis implies that observed distinct sizes for the doughnut intensity profiles in figure 4(b) is due to nonzero curvature of the incident Gaussian beam in the plane of the sample.

Above two examples emphasize the role of the propagation-induced wavefront curvature before or after the sample, respectively. These results recall that any practical deviation from an ideal experimental implementation can bring substantial swirl-induced symmetry breaking. This leads us to the next section where we propose an attempt to control the very existence of the swirl.

\section{Taming the swirl}

\subsection{Experimental approach}

Starting from the fact that the occurrence of the swirled UQPs cannot the avoided (figure 1) we propose a compensation scheme to get rid of it owing to the arithmetic of geometric phase optical elements. Latter has already received attention in the context of generating phase singularities with topological charges that differs from $\pm 2 q[27,28]$ or passive tuning the retardance of $q$-plates [29]. In all cases, an effective behavior is obtained by an appropriate combination of $q$-plates and homogeneous optical retarders. Here we extend these approaches to the case of radially inhomogeneous elements, the basic idea being to cancel the swirl of a first UQP by that of a second UQP having opposite swirl.

The setup used to implement this compensation strategy is shown in figure 6, where the afocal system made of the pair of lenses inserted between $\mathrm{UQP}_{1}$ and $\mathrm{UQP}_{2}$ allows one-to-one superposition of their respective swirls while preserving the incident collimation. Two configurations, which respectively correspond to UQPs retardance set to half-wave and quarterwave conditions, are detailed in the next subsections. 


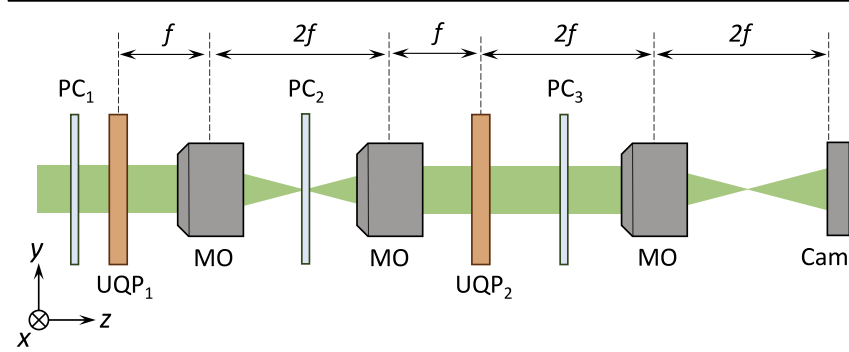

Figure 6. Setup for observing the effective phase mask resulting from swirl arithmetics with two cascaded elements, $\mathrm{UQP}_{1}$ and $\mathrm{UQP}_{2}$. MO: microscope objective with $4 \times$ magnification, numerical aperture $\mathrm{NA}=0.1$ and effective focal length $f=45 \mathrm{~mm}$.
$\mathrm{UQP}_{1}$

(a)

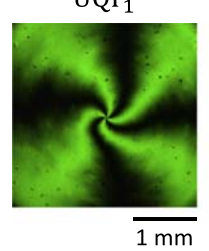
$\mathrm{UQP}_{1}$

(b)

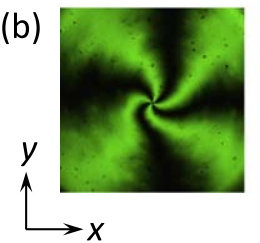

$\mathrm{UQP}_{2}$
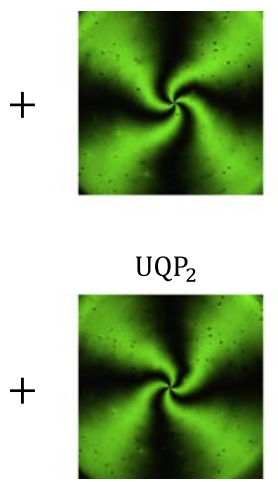
$=$
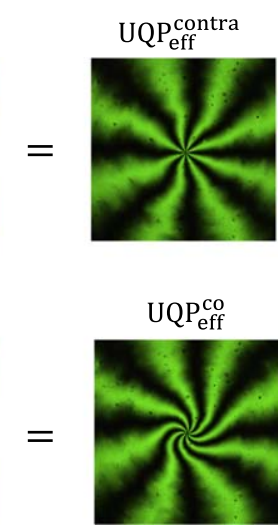

Figure 7. Half-wave cascading scheme. Individual and effective UQPs observed between crossed linear polarizers using incoherent illumination using the setup shown in figure 6 for $\zeta_{1}=-\zeta_{2}$ (a) and $\zeta_{1}=\zeta_{2}$ (b). Here, $\zeta_{1}=+1$. The observation of $\mathrm{UQP}_{1}$ (resp. $\mathrm{UQP}_{2}$ ) alone is made by setting $\left(U_{1}, U_{2}\right)=(2.60,3.00) \mathrm{V}$ (resp. $\left(U_{1}, U_{2}\right)=$ $(3.00,2.60) \mathrm{V})$ noting that an applied voltage of $3.00 \mathrm{~V}$ corresponds to neutral $2 \pi$ birefringent phase retardation; see figure 3(a).

\subsection{Half-wave configuration}

Assuming an incident circularly polarized light with helicity $\sigma$, the half-wave retardance setting lead to impart a phase profile $\Phi_{1}=2 \sigma\left[\phi+\zeta_{1} \varphi(r)\right]$ to the light emerging from $\mathrm{UQP}_{1}$, which has a helicity $-\sigma$. Then, the helicity is flipped from $-\sigma$ to $\sigma$ owing to the polarization controller $\mathrm{PC}_{2}$ tuned to half-wave retardance. Further, $\mathrm{UQP}_{2}$ imparts a phase profile $\Phi_{2}=2 \sigma\left[\phi+\zeta_{2} \varphi(r)\right]$ to the light passing through it. Overall, the cascaded UQPs behave as an effective one imparting a phase equal to the sum of the contributions from the two UQPs. Namely

$$
\Phi_{\mathrm{eff}}=4 \sigma \phi+2 \sigma\left(\zeta_{1}+\zeta_{2}\right) \varphi(r)
$$

to the incident light. The individual swirls cancel one another out for contra-swirled UQPs $\left(\zeta_{1}=-\zeta_{2}\right)$ while they add up for co-swirled UQPs $\left(\zeta_{1}=\zeta_{2}\right)$. The experimental demonstration is shown in figure 7 that shows the image of the effective birefringent phase masks observed between crossed linear polarizers for contra-swirled (figure 7(a)) and co-swirled (figure 7(b)) geometries. The swirl is actually either canceled or doubled, whereas the topological charge of the effective
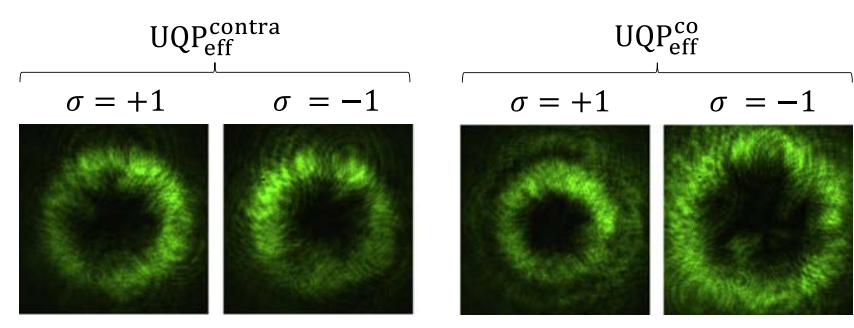

Figure 8. Far-field intensity profiles of the light beam passing through the contra-swirled $\left(\zeta_{1}=-\zeta_{2}\right)$ or co-swirled $\left(\zeta_{1}=\zeta_{2}\right)$ cascaded UQPs. The corresponding experimental scheme is described in figure 4(a). The incident collimated Gaussian beam has circular polarization with helicity $\sigma$. (a)
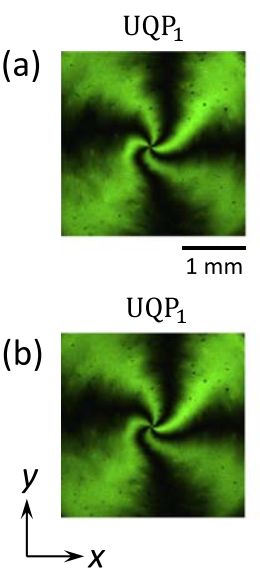
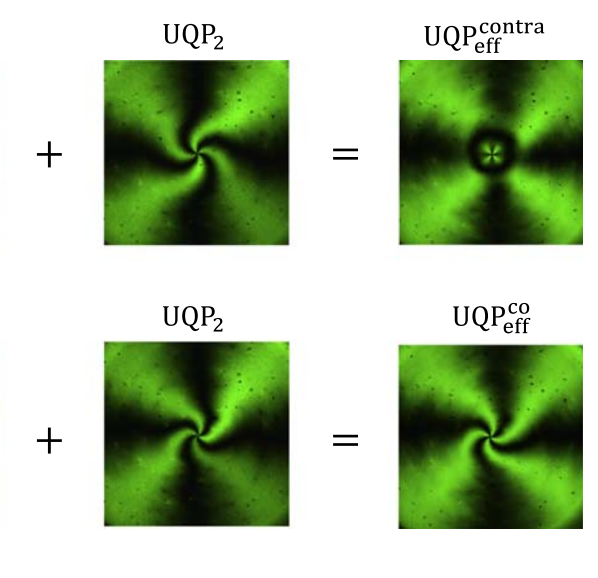

Figure 9. Quarter-wave cascading scheme. The presentation is similar to that of figure 7 . The observation of $\mathrm{UQP}_{1}$ (resp. $\mathrm{UQP}_{2}$ ) alone is made by setting $\left(U_{1}, U_{2}\right)=(2.42,3.00) \mathrm{V}$ (resp. $\left.\left(U_{1}, U_{2}\right)=(3.00,2.42) \mathrm{V}\right)$.

UQP is $q_{\text {eff }}=+2$ in both cases, as one can judge from visual inspection of the eight-brush patterns.

Moreover, we checked the sensitivity to residual curvature effects observed in far-field discussed in section 3 but this time for two cascaded UQPs. As expected, the swirl-provided discriminatory lensing vanishes in the contra-swirled situation, whereas it is enhanced for co-swirled individual UQPs; see the images in figure 8 . In the latter figure, the observed ringing intensity profile in the third panel recalls the fact that we are dealing with a superposition of Laguerre-Gauss modes, even in the absence of the swirl [30], and that curvature effects are also present. Also, we have evaluated the ratio between the radii of maximal intensity of the far-field intensity similarly to the single-mask case and the results are shown in figure 5 as dashed curves.

\subsection{Quarter-wave configuration}

As noticed above, the half-wave configuration doubles the topological charge, hence raising the question whether one could cancel the swirl while preserving an effective UQP with $q_{\text {eff }}=+1$. This leads us to consider a cascade of two UQPs each set to a quarter-wave configuration. In this case, the $\mathrm{PC}_{2}$ placed between the two UQPs should be removed. The results of such combination are shown in figure 9. As expected, the co-swirled geometry (b) does not bring added-value since the 
obtained effective UQP qualitatively corresponds to a single UQP set to half-wave retardance. In contrast, although the contra-swirled geometry (a) actually leads to a non-swirled effective structure, it does not correspond anymore to an effective geometric phase optical element characterized by a spatial distribution of the optical axis orientation angle, $\psi_{\text {eff }}(r, \phi)$, and a spatial distribution of the birefringent phase retardation, $\Delta_{\text {eff }}(r, \phi)$.

Indeed, let us recall the expression of the Jones matrix $\boldsymbol{J}$ of a geometric phase optical element made of a $z$-invariant anisotropic and inhomogeneous slab fully characterized by the arbitrary distributions $\psi(r, \phi)$ and $\Delta(r, \phi)$. In the circular polarization basis $\left(\mathbf{c}_{+}, \mathbf{c}_{-}\right)$associated with the helicity $(+1,-1)$, we have

$$
J(\Delta, \psi)=\left(\begin{array}{cc}
\cos \frac{\Delta}{2} & i \sin \frac{\Delta}{2} e^{-2 i \psi} \\
i \sin \frac{\Delta}{2} e^{2 i \psi} & \cos \frac{\Delta}{2}
\end{array}\right)
$$

The effective Jones matrix in the contra-swirled quarterwave configuration is, taking $\zeta_{1}=-\zeta_{2}=\zeta$,

$$
\mathbf{J}_{\mathrm{eff}}=\mathbf{J}(\pi / 2, \phi-\zeta \varphi(r)) \mathbf{J}(\pi / 2, \phi+\zeta \varphi(r)) \text {. }
$$

The latter expression simplifies to

$$
J_{\mathrm{eff}}=\frac{1}{2}\left(\begin{array}{cc}
1-e^{4 i \zeta \varphi(r)} & 2 i \cosh (2 i \zeta \varphi(r)) e^{-2 i \phi} \\
2 i \cosh (2 i \zeta \varphi(r)) e^{2 i \phi} & 1-e^{-4 i \zeta \varphi(r)}
\end{array}\right),
$$

which cannot be recast in the form given by equation (7) for $\varphi(r) \neq 0$. As such, this effective optical element is not useful when one needs to generate usual optical vortex beams carrying on-axis phase singularity.

\section{Conclusion}

Summarizing, we have reported on the quantitative analysis of unavoidably swirled in-plane distortions of self-structured UQPs in the framework of a magneto-electric excitation. In particular, we pointed out that the helicity-dependent radial dependence of the geometric phase associated to the swirl can affect substantially the spatial distribution of light in experiments as soon as residual curvature effects are present. Moreover, we have demonstrated that existence of the swirl can be controlled in an effective manner by cascading two swirled elements in order to either cancel or enhance aspheric geometric phase lensing induced by the swirl. The present study also invites further experimental work to unravel a possible swirl in the third dimension and its effects on light. Therefore, recalling that the umbilical defects at work here have only been described so far within two-dimensional mathematical treatments [31, 22], theoretical efforts to assess the three-dimensional nature of umbilics are also worth considering.

\section{ORCID iDs}

Etienne Brasselet (ib https://orcid.org/0000-0001-6672-6785

\section{References}

[1] Allen L, Beijersbergen M W, Spreeuw R J C and Woerdman J P 1992 Orbital angular momentum of light and the transformation of Laguerre-Gaussian laser modes Phys. Rev. A $\mathbf{4 5} 8185$

[2] Hefner B T and Marston P L 1999 An acoustical helicoidal wave transducer with applications for the alignment of ultrasonic and underwater systems J. Acoust. Soc. Am. $1063313-6$

[3] Bliokh K Y, Bliokh Y P, Savel'Ev S and Nori F 2007 Semiclassical dynamics of electron wave packet states with phase vortices Phys. Rev. Lett. 99190404

[4] Bryngdahl O 1973 Radial-and circular-fringe interferograms J. Opt. Soc. Am. 63 1098-104

[5] Khonina S, Kotlyar V, Shinkaryev M, Soifer V and Uspleniev G 1992 The phase rotor filter J. Mod. Opt. 39 1147-54

[6] Bhandari R 1997 Polarization of light and topological phases Phys. Rep. 281 1-64

[7] Bliokh K Y, Rodríguez-Fortuño F J, Nori F and Zayats A V 2015 Spin-orbit interactions of light Nat. Photon. 9796

[8] Marrucci L, Manzo C and Paparo D 2006 Optical spin-toorbital angular momentum conversion in inhomogeneous anisotropic media Phys. Rev. Lett. 96163905

[9] Rubano A, Cardano F, Piccirillo B and Marrucci L 2019 Qplate technology: a progress review J. Opt. Soc. Am. B 36 D70-87

[10] Piccirillo B, D'Ambrosio V, Slussarenko S, Marrucci L and Santamato E 2010 Photon spin-to-orbital angular momentum conversion via an electrically tunable q-plate Appl. Phys. Lett. 97241104

[11] Serabyn E, Prada C M, Chen P and Mawet D 2019 Vector vortex coronagraphy for exoplanet detection with spatially variant diffractive waveplates J. Opt. Soc. Am. B 36 D13-9

[12] Brasselet E, Murazawa N, Misawa H and Juodkazis S 2009 Optical vortices from liquid crystal droplets Phys. Rev. Lett. 103103903

[13] Brasselet E and Loussert C 2011 Electrically controlled topological defects in liquid crystals as tunable spin-orbit encoders for photons Opt. Lett. 36 719-21

[14] Loussert C, Delabre U and Brasselet E 2013 Manipulating the orbital angular momentum of light at the micron scale with nematic disclinations in a liquid crystal film Phys. Rev. Lett. 111037802

[15] Loussert C, Kushnir K and Brasselet E 2014 Q-plates microarrays for parallel processing of the photon orbital angular momentum Appl. Phys. Lett. 105121108

[16] Barboza R, Bortolozzo U, Assanto G, Vidal-Henriquez E, Clerc M G and Residori S 2012 Vortex induction via anisotropy stabilized light-matter interaction Phys. Rev. Lett. 109143901

[17] Kravets N, Podoliak N, Kaczmarek M and Brasselet E 2019 Self-induced liquid crystal q-plate by photoelectric interface activation Appl. Phys. Lett. 114061101

[18] Brasselet E 2018 Tunable high-resolution macroscopic selfengineered geometric phase optical elements Phys. Rev. Lett. 121033901

[19] Ghadimi Nassiri M and Brasselet E 2018 Multispectral management of the photon orbital angular momentum Phys. Rev. Lett. 121213901

[20] Kravets N and Brasselet E 2018 Nonlinear unitary transformations of space-variant polarized light fields from self-induced geometric-phase optical elements Phys. Rev. A 97013834

[21] Barboza R, Bortolozzo U, Clerc M G, Residori S and Vidal-Henriquez E 2014 Light-matter interaction induces a single positive vortex with swirling arms Phil. Trans. R. Soc. A 3722014 
[22] Clerc M G, Vidal-Henriquez E, Davila J D and Kowalczyk M 2014 Symmetry breaking of nematic umbilical defects through an amplitude equation Phys. Rev. E 90012507

[23] Tortora L and Lavrentovich O D 2011 Chiral symmetry breaking by spatial confinement in tactoidal droplets of lyotropic chromonic liquid crystals Proc. Natl Acad. Sci. 108 5163-8

[24] Tam A M et al 2017 Bifocal optical-vortex lens with sorting of the generated nonseparable spin-orbital angular-momentum states Phys. Rev. Appl. 7034010

[25] Aleksanyan A, Kravets N and Brasselet E 2017 Multiple-star system adaptive vortex coronagraphy using a liquid crystal light valve Phys. Rev. Lett. 118203902

[26] Collins S A 1970 Lens-system diffraction integral written in terms of matrix optics J. Opt. Soc. Am. 60 1168-77
[27] Marrucci L, Manzo C and Paparo D 2006 Pancharatnam-berry phase optical elements for wave front shaping in the visible domain: switchable helical mode generation Appl. Phys. Lett. 88221102

[28] Delaney S, Sánchez-López M M, Moreno I and Davis J A 2017 Arithmetic with q-plates Appl. Opt. 56 596-600

[29] Radhakrishna B, Kadiri G and Raghavan G 2019 Wavelengthadaptable effective q-plates with passively tunable retardance Sci. Rep. 9 1-9

[30] Vallone G, Sponselli A, V D'ambrosio, Marrucci L, Sciarrino F and Villoresi P 2016 Birth and evolution of an optical vortex Opt. Express 2416390

[31] Rapini A 1973 Umbilics: Static properties and shear-induced displacements J. Phys 34 629-33 